\title{
Versão curta do Inventário de Perspectiva Temporal de Zimbardo (ZTPI-25):
}

\section{validação brasileira}

\author{
Short version of the Zimbardo Time Perspective Inventory (ZTPI-25): Brazilian validation \\ Versión corta del Inventario de Perspectiva Temporal de Zimbardo (ZTPI-25): validación \\ brasileña
}

Recebido: 08/02/2021 | Revisado: 12/02/2021 | Aceito: 15/02/2021 | Publicado: 22/02/2021

Hellen Pollyanna Mantelo Cecilio
ORCID: https://orcid.org/0000-0002-6597-432X
Universidade Estadual de Maringá, Brasil
E-mail: pollymantelo@ gmail.com
Thémistoklis Apostolidis
ORCID: https://orcid.org/0000-0002-3549-5547
Aix-Marseille Université, França
E-mail: themistoklis.apostolidis@ univ-amu.fr
Dimitrios Lampropoulos
ORCID: https://orcid.org/0000-0001-9505-5650
Aix-Marseille Université, França
E-mail: dimilamprop@ gmail.com
Denize Cristina de Oliveira
ORCID: https://orcid.org/0000-0002-0830-0935
Universidade do Estado do Rio de Janeiro, Brasil
E-mail: dcouerj@ gmail.com

\section{Resumo}

Objetivo: investigar as evidências de validade (de construto, estrutural e convergente) de uma versão curta do Inventário de Perspectiva Temporal de Zimbardo para a população brasileira. Método: os dados foram coletados com 281 pessoas vivendo com HIV, atendidas em serviços públicos de saúde. Realizou-se análise fatorial exploratória, análise fatorial confirmatória e correlações, resultando na proposição de uma versão curta contendo 25 itens. Resultados: identificou-se o ZTPI-25 como melhor opção para a população brasileira, apresentando ajuste satisfatório, preservação das propriedades psicométricas e premissas teóricas do instrumento original. A validade estrutural foi conferida pela homogeneidade entre os fatores e a validade convergente foi admitida pelas correlações entre os construtos, que se apresentaram significativas. Conclusão: O ZTPI-25 conserva as propriedades psicométricas da versão original e apresenta como vantagens o menor tempo de aplicação, menor índice de rejeição e redução de questões sem respostas, quando comparado às outras escalas validadas no Brasil.

Palavras-chave: Perspectiva temporal; Percepção do tempo; Psicometria; Estudos de validação.

\begin{abstract}
Objective: to investigate the evidence of validity (of construct, structural and convergent) of a short version of the Zimbardo Temporary Perspective Inventory for the Brazilian population. Method: data were collected from 281 people living with HIV, seen at public health services. Exploratory factor analysis, confirmatory factor analysis and correlations were performed, resulting in the proposition of a short version containing 25 items. Results: ZTPI-25 was identified as the best option for the Brazilian population, with satisfactory adjustment, preservation of psychometric properties and theoretical premises of the original instrument. The structural validity was conferred by the homogeneity between the factors and the convergent validity was admitted by the correlations between the constructs, which presented significant. Conclusion: The ZTPI-25 preserves the psychometric properties of the original version, and as having the advantages of shorter application times, lower rejection rates and reduced unanswered questions when compared to other scales validated in Brazil.
\end{abstract}

Keywords: Time perspective; Time perception; Psychometrics; Validation studies.

\section{Resumen}

Objetivo: investigar la evidencia de validez (de constructo, estructural y convergente) de una versión corta del Inventario de Perspectivas Temporales de Zimbardo para la población brasileña. Método: se recopilaron datos de 281 personas que viven con el VIH, atendidas en servicios de salud pública. Se realizaron análisis factoriales exploratorios, análisis factoriales confirmatorios y correlaciones, que resultó en propuesta de una versión corta que contenía 25 ítems. Resultados: ZTPI-25 fue identificada como la mejor opción para población brasileña, con ajuste 
satisfactorio, preservación de propiedades psicométricas y premisas teóricas del instrumento original. La validez estructural fue conferida por la homogeneidad entre los factores y la validez convergente fue admitida por correlaciones entre las construcciones, que se presentaron significativas. Conclusión: ZTPI-25 conserva las propiedades psicométricas de versión original, ventajas de tiempos de aplicación más cortos, tasas de rechazo más bajas y preguntas sin respuesta reducidas en comparación con otras escalas validadas en Brasil.

Palabras clave: Perspectiva del tiempo; Percepción del tiempo; Psicometría; Estudios de validación.

\section{Introdução}

A percepção do tempo se constitui como uma capacidade inata do ser humano que define sua forma de perceber o mundo, sendo uma experiência individual e subjetiva. Reconhece-se que "o passado e o futuro exercem influência no comportamento presente, em que há uma trama de pontos de vista individuais e psicológicos do futuro e do passado existentes em determinado momento" (Lewin, 1951, p.75). Assim, o tempo é essencial para a condução da vida humana sendo inerente ao indivíduo.

A noção de perspectiva temporal disseminou-se em estudos no exterior, no entanto, ainda é pouco explorada no Brasil, sendo definida como a forma como o indivíduo encara o tempo, pressupondo que em um dado momento - presente psicológico - podem coexistir o passado e o futuro psicológicos. Considera-se que o tempo desempenha papel importante no modo como as pessoas atribuem sentido e se posicionam diante da vida, dando coerência e continuidade a mesma (Lewin, 1951; Oliveira, Cecilio \& Oliveira, 2020).

A Teoria da Perspectiva Temporal foi concebida em três dimensões do tempo psicológico: perspectiva temporal (PT), atitude temporal e orientação temporal. A perspectiva temporal se caracteriza pela distância temporal dos objetos imaginados, densidade com que os objetos se distribuem pelas regiões temporais, existência ou não de relações entre os objetos e nível de realismo com que os objetos são percebidos pelos indivíduos. A atitude temporal se caracteriza pela valência positiva ou negativa atribuída aos acontecimentos, pela maior ou menor satisfação com o passado ou presente e pelo grau de otimismo em relação ao futuro. A orientação temporal compreende a direção predominante do pensamento e do comportamento de um indivíduo em relação a acontecimentos do passado, presente ou futuro (Nuttin \& Lens, 1985).

A perspectiva temporal tem sido considerada como contexto primário a partir do qual os seres humanos entendem e dão sentido as suas experiências de vida, uma das forças cruciais na vida das pessoas e desempenha influência nos comportamentos, atitudes e na tomada de decisões (Oliveira, Cecilio \& Oliveira, 2020). No contexto da Psicologia do Tempo, a perspectiva temporal é definida como "um processo, muitas vezes inconsciente, pelo qual os fluxos contínuos de experiências pessoais e sociais são atribuídos às categorias temporais, que ajudam a dar ordem, coerência e significado a esses eventos" (Zimbardo \& Boyd, 1999, p.1271).

Estudos apontam que a perspectiva temporal se manifesta de forma distinta ao longo da vida e atua como um forte motivador para práticas de saúde positivas e auxilia na compreensão das relações que favorecem a manutenção de comportamentos saudáveis ou de risco, tanto mental quanto físico (Cecilio et al., 2020; Leite \& Pasquali, 2008; Roncancio, Ward \& Fernandez, 2014; Zimbardo \& Boyd, 1999). Comumente, o indivíduo não tem discernimento de tal influência, assim, ao converter a perspectiva temporal em algo consciente, ela passa a ser controlada e, ao permitir esse feito, poderá conduzir a vida de forma mais feliz e bem sucedida (Zimbardo \& Boyd, 1999).

Destarte, a perspectiva temporal mostra-se particularmente importante para as pessoas vivendo com HIV, uma vez que em situação de adoecimento de longo prazo, observar o passado e planejar o futuro são essenciais para assegurar um presente mais estável, tanto no que se refere à saúde física quanto psicológica e social. Assim, a tomada de decisão poderá ser influenciada pela dimensão temporal dominante, ou seja, algumas pessoas precisam recordar situações do passado e avaliar seus custos e benefícios para as decisões atuais, de modo que tais recordações podem ser traumáticas, negativas ou ainda, nostálgicas e positivas (Cecilio et al., 2020; Oliveira, Cecilio \& Oliveira, 2020). 
Ao considerar a perspectiva temporal como um construto da psicologia que analisa a orientação temporal dos indivíduos e pode ter influência no comportamento, o campo da saúde pode se beneficiar da sua aplicação, a partir da explicação do porque as pessoas têm dificuldades em manter padrões de comportamentos saudáveis, ao mesmo modo que mantém outros, que levam ao adoecimento de natureza mental e física (Oliveira, Cecilio \& Oliveira, 2020).

A perspectiva temporal pode ser um marcador de vulnerabilidades relacionadas à saúde, uma vez que se refere à orientação temporal relativa que motiva as ações e metas típicas de um indivíduo ou de um grupo. Desse modo, o construto da perspectiva temporal permite que os profissionais de saúde busquem estratégias mais holísticas para a gestão de doenças crônicas centradas no indivíduo. Nesse sentido, infere-se que a integração da avaliação da perspectiva temporal nas práticas do cuidado poderá auxiliar na investigação de comportamentos e atitudes, que por ventura interfiram nos processos de autocuidado (Oliveira, Cecilio \& Oliveira, 2020).

Acredita-se na importância de se considerar a perspectiva temporal nas práticas de cuidado de enfermagem, tanto para prevenção de comportamentos danosos à saúde, como para o desenvolvimento de perspectivas incentivadoras de comportamentos protetores à saúde. Considera-se a perspectiva temporal como uma característica passível de modificação, contudo, reitera-se a necessidade de estudos adicionais sobre a estabilidade e a mutabilidade do construto (Oliveira, Cecilio \& Oliveira, 2020).

Considerando a importância da perspectiva temporal e a necessidade de uma ferramenta que permita a aferição consistente do construto, o objetivo deste estudo foi investigar as evidências de validade (de construto, estrutural e convergente) de uma versão curta do Inventário de Perspectiva Temporal de Zimbardo para a população brasileira.

\section{Metodologia}

Adotou-se amostragem não-probabilística, de conveniência, escolhida a partir de informações do quantitativo de pessoas em seguimento em cada unidade de saúde, disponibilizadas por um Serviço de Atendimento Especializado em HIV/AIDS em Niterói e em três Centros Municipais de Saúde no Rio de Janeiro. Os critérios de inclusão dos participantes foram: sorologia positiva para HIV; idade maior ou igual a 18 anos; estar em condições clínicas e psicológicas para responder aos instrumentos; estar em acompanhamento no serviço selecionado. Como critério de exclusão, os indivíduos que se declaravam analfabetos, com deficiência visual importante ou falta de condições clínicas foram substituídos.

Os indivíduos foram convidados a participar conforme a sua presença nos serviços de saúde nos períodos de coleta dos dados, até completar o número definido para a amostra em cada município, procedendo-se a substituição em caso de recusa ou desistência. A amostra estudada considerou a premissa que na análise fatorial são necessários pelo menos cinco participantes por variável e uma amostra total de pelo menos 200 sujeitos (Laros, 2012), sendo assim, a amostra foi composta por 281 pessoas vivendo com HIV, 101 de Niterói e 180 do Rio de Janeiro. Os instrumentos foram autoaplicados, sendo orientado aos participantes assinalar a opção que melhor se adequava a sua vida. A coleta de dados foi realizada entre março e outubro de 2016. A pesquisa foi desenvolvida conforme a Resolução 466/12 do Conselho Nacional de Saúde e obteve todas as aprovações pertinentes (Pareceres 1.341.344/2015 e 1.441.788/2016). O Termo de Consentimento Livre e Esclarecido foi disponibilizado a todos os participantes, na ocasião do primeiro contato, garantindo sigilo e anonimato.

Para análise da qualidade de vida, utilizou-se o instrumento WHOQOL-HIV bref, contendo 31 questões, distribuídas em seis domínios (físico, psicológico, nível de independência, relações sociais, meio ambiente e espiritualidade, religião e crenças pessoais), em escala Likert, para estimar aspectos da vida das pessoas que vivem com o vírus (Zimpel \& Fleck, 2007). O cálculo dos escores da qualidade de vida foi realizado por meio de média simples, agrupando as questões correspondentes a cada domínio (OMS, 2002).

Utilizou-se um questionário, construído para esta pesquisa, contendo 24 variáveis relativas às características 
socioeconômicas e clínicas dos participantes. Os dados socioeconômicos, clínicos e de qualidade de vida foram utilizados como suporte para a validade do ZTPI-25, a fim de comparação com análises anteriormente realizadas (Laguette et al., 2013; Préau et al., 2007). A análise foi realizada com estatística descritiva e inferencial.

Os dados da perspectiva temporal foram analisados seguindo as proposições da análise original (Zimbardo \& Boyd, 1999). O Inventário de Perspectiva Temporal de Zimbardo (ZTPI) foi construído a partir de uma base teórica definida, considerando processos sociais, cognitivos e motivacionais, com o objetivo de avaliar a perspectiva temporal de uma forma global, abarcando três zonas de orientação do tempo: passado, presente e futuro. O inventário apresenta 56 itens, dispostos em escala Likert de cinco pontos, em cinco dimensões: passado-negativo; passado-positivo; presente-fatalista; presente-hedonista; e futuro (Zimbardo \& Boyd, 1999). As validações do ZTPI no Brasil (Leite \& Pasquali, 2008; Milfont et al., 2008) e no exterior (Apostolidis \& Fieulaine, 2004; Davis \& Ortiz, 2017; Roncancio, Ward \& Fernandez, 2014) apresentam divergências entre os processos e métodos utilizados, com diferentes subescalas e padrões de análise, o que contribuiu para o questionamento da qualidade das estruturas apresentadas. Ainda, identificou-se uma tendência a utilizar versões curtas do ZTPI em diversos estudos (Košt'ál et al., 2016; Orkibi, 2015; Ortuño et al., 2017; Sircova et al., 2014; Zhang, Howell \& Bowerman, 2013).

A evidência de validade de construto foi observada pela Análise Fatorial Exploratória (AFE) e Análise Fatorial Confirmatória (AFC). A AFE, para redução da complexidade das variáveis (Figueiredo \& Silva, 2010), foi realizada por meio da Análise de Componentes Principais, com rotação Varimax, fixando a extração em cinco fatores e substituindo possíveis valores ausentes pela média da variável e extração de carga fatorial $\geq 0,30$, conforme análise original (Zimbardo \& Boyd, 1999). Considerou-se a adequação da amostra e o método pertinente quando o teste KMO (medida Kaiser-Meyer-Olkin de adequação) $\geq 0,70$ e o teste de esfericidade de Bartlett significativo (Figueiredo \& Silva, 2010).

A análise dos modelos por meio da AFC considerou, primeiramente, o valor do índice RMSEA (root mean square error of approximation), que considera um bom ajustamento valores próximos a 0,05; em segundo lugar, o valor do quiquadrado por graus de liberdade $\left(\chi^{2} / \mathrm{gl}\right)$, que menor de dois, significa melhor ajustamento; em terceiro, o índice CFI (comparative fit índex), indica melhor resultado acima de 0,90; e em quarto a facilidade de convergência da solução (número de iterações), que sinaliza que a solução da variável latente converge, isto é, quando os valores iniciais são semelhantes às estimativas de parâmetros dos valores do modelo final, são necessárias menos iterações para a convergência (Apostolidis \& Fieulaine, 2004; Brown, 2006).

Para testar a confiabilidade utilizou-se o Alfa de Cronbach $(\alpha)$, considerando boa consistência interna quando valores foram $\geq 0,70$ (Brown, 2006). Foi realizada AFC para a obtenção da validade de construto, testando cinco modelos sendo, Modelo 1: versão original (Zimbardo \& Boyd, 1999); Modelo 2: versão adaptada validada no Brasil (Leite \& Pasquali, 2008); Modelo 3: versão com 38 itens validada no Brasil (Milfont et al., 2008) e as estruturas propostas neste estudo, Modelo 4: versão curta com 15 itens e Modelo 5: versão curta com 25 itens. As duas versões curtas propostas foram constituídas a partir da seleção dos três itens com maiores pontuações de cada subescala para compor a versão com 15 itens; e dos cinco itens com maior pontuação em cada subescala para compor a versão com 25 itens. Foram construídos cinco diagramas, um para cada modelo testado.

Após a confirmação de adequação do modelo, realizou-se intercorrelações das subescalas a fim de garantir a validade estrutural. A validade convergente foi verificada por meio da correlação de Pearson entre as subescalas da versão curta do ZTPI, dados socioeconômicos e clínicos e os domínios da qualidade de vida. Para análise, foi utilizado o software SPSS ${ }^{\circledR}$, versão 20, com pacote AMOS Graphics ${ }^{\circledR}$. Para todas as análises considerou-se estatisticamente significativo quando $\mathrm{p} \leq 0,05$. 


\section{Resultados}

Os participantes caracterizaram-se por: 68,7\% do sexo masculino; idade entre 18 e 72 anos (média: 41,1 anos; desviopadrão $\pm 12,7)$; ensino médio (57,7\%); em utilização de terapia antirretroviral (94,3\%); sem sintomas relacionados ao HIV (89,7\%). O teste KMO resultou em 0,77, enquanto o teste de esfericidade de Bartlett apontou significância com p<0,01. A solução encontrada explicou 34,4\% da variância do instrumento, com a maior carga fatorial de 0,74. Dos 56 itens originais do ZTPI, três $(33,47,55)$ não atingiram carga fatorial mínima de 0,30 e alguns itens saturaram em mais de um componente.

A proposição de duas escalas reduzidas foi realizada a partir dos resultados da matriz rotativa dos componentes e das cargas fatoriais. Selecionaram-se os três itens com maior carga fatorial em cada subescala para compor a versão do ZTPI com 15 itens, contemplando passado-negativo, itens 50, 54 e 16; passado-positivo, itens 2, 7 e 29; presente-fatalista, itens 39, 37 e 24; presente-hedonista, itens 8, 23 e 42; futuro, itens 56, 40 e 10. Posteriormente, selecionaram-se os cinco itens com maior carga fatorial em cada subescala, para compor a versão do ZTPI com 25 itens: passado-negativo, itens 50, 54, 16, 34 e 4; passado-positivo, itens 2, 7, 29, 20 e 11; presente-fatalista, itens 39, 37, 24, 28 e 32; presente-hedonista, itens 8, 23, 42, 53 e 31; futuro, itens 56, 40, 10, 45 e 21. Essas duas escalas reduzidas foram testadas, a fim de garantir propriedades psicométricas confiáveis.

Os resultados da comparação das subescalas dos cinco modelos analisados em termos de médias, desvio-padrão e Alfa de Cronbach estão apresentados na Tabela 1.

Tabela 1 - Média, desvio-padrão (dp) e Alfa de Cronbach das subescalas do ZTPI, Rio de Janeiro e Niterói, 2018.

\begin{tabular}{llllll}
\hline & $\mathrm{PN}^{*}$ & $\mathrm{PP}^{*}$ & $\mathrm{PF}^{*}$ & $\mathrm{PH}^{*}$ & $\mathrm{~F}^{*}$ \\
\hline Modelo 1 & $\mathbf{1 0}$ itens & $\mathbf{9}$ itens & $\mathbf{9}$ itens & $\mathbf{1 5}$ itens & $\mathbf{1 3}$ itens \\
Média $\pm \mathrm{dp}$ & $3,02 \pm 0,81$ & $3,44 \pm 0,66$ & $2,95 \pm 0,66$ & $3,32 \pm 0,60$ & $3,45 \pm 0,45$ \\
Alfa $(\alpha)$ & 0,80 & 0,63 & 0,71 & 0,75 & 0,47 \\
Modelo 2 & $\mathbf{1 3}$ itens & $\mathbf{1 0}$ itens & $\mathbf{9}$ itens & $\mathbf{1 3}$ itens & $\mathbf{1 3}$ itens \\
Média \pm dp & $3,06 \pm 0,69$ & $3,36 \pm 0,65$ & $3,10 \pm 0,78$ & $3,26 \pm 0,63$ & $3,55 \pm 0,50$ \\
Alfa $(\alpha)$ & 0,76 & 0,66 & 0,74 & 0,72 & 0,56 \\
Modelo 3 & $\mathbf{7}$ itens & $\mathbf{6}$ itens & $\mathbf{6}$ itens & $\mathbf{9}$ itens & $\mathbf{1 0}$ itens \\
Média \pm dp & $3,12 \pm 0,81$ & $3,54 \pm 0,77$ & $2,89 \pm 0,83$ & $3,26 \pm 0,66$ & $3,61 \pm 0,53$ \\
Alfa $(\alpha)$ & 0,70 & 0,65 & 0,62 & 0,63 & 0,52 \\
Modelo 4 & $\mathbf{3}$ itens & $\mathbf{3}$ itens & $\mathbf{3}$ itens & $\mathbf{3}$ itens & $\mathbf{3}$ itens \\
Média $\pm d p$ & $3,00 \pm 1,16$ & $3,41 \pm 1,01$ & $3,01 \pm 1,04$ & $2,75 \pm 1,03$ & $3,82 \pm 0,82$ \\
Alfa $(\alpha)$ & 0,75 & 0,61 & 0,62 & 0,64 & 0,59 \\
Modelo 5 & $\mathbf{5}$ itens & $\mathbf{5}$ itens & $\mathbf{5}$ itens & $\mathbf{5}$ itens & $\mathbf{5}$ itens \\
Média \pm dp & $3,08 \pm 1,04$ & $3,56 \pm 0,86$ & $2,93 \pm 0,91$ & $2,75 \pm 0,85$ & $3,85 \pm 0,72$ \\
Alfa $(\alpha)$ & 0,79 & 0,70 & 0,69 & 0,64 & 0,64 \\
\hline
\end{tabular}

${ }^{*}$ PN: passado-negativo; PP: passado-positivo; PF: presente-fatalista; PH: presente-hedonista; F: futuro. Fonte: Autores (2018).

Observa-se na Tabela 1 que as médias das subescalas são semelhantes para os diferentes modelos analisados, porém, os valores do Alfa nos Modelos 4 e 5, versões reduzidas, são mais próximos aos do Modelo 1 do que as demais validações brasileiras. A Tabela 2 mostra os índices para a comparação dos cinco modelos. 
Tabela 2 - Índices dos modelos testados do ZTPI, Rio de Janeiro e Niterói, 2018.

\begin{tabular}{lcccccc}
\hline & $\chi^{2}$ & $\mathrm{gl}$ & $\chi^{2} / \mathrm{gl}$ & RMSEA & CFI & Iterações \\
\hline Modelo 1 & 3067,14 & 1474 & 2,08 & 0,06 & 0,55 & 17 \\
Modelo 2 & 2347,80 & 1157 & 2,02 & 0,06 & 0,61 & 13 \\
Modelo 3 & 1392,09 & 655 & 2,12 & 0,06 & 0,60 & 12 \\
Modelo 4 & 171,970 & 80 & 2,13 & 0,06 & 0,88 & 12 \\
Modelo 5 & 512,578 & 265 & 1,93 & 0,05 & 0,82 & 8 \\
\hline
\end{tabular}

Fonte: Autores (2018).

A partir dos resultados apresentados na Tabela 2, identificou-se que o Modelo 5 apresentou o índice RMSEA e taxa $\chi^{2} / \mathrm{gl}$ adequados, além do menor número de iterações, demonstrando um ajuste satisfatório. Embora o CFI não tenha atingido valor esperado $(0,90)$ em nenhum modelo, o Modelo 5 alcançou o segundo melhor índice $(0,82)$.

Adotando-se o Modelo 5 - ZTPI-25 - como a melhor versão, procederam-se as demais análises. Realizou-se a AFE com os 25 itens, rotação Varimax e extração fixada em cinco componentes, explicando 49,03\%. Assim, os componentes foram: passado-negativo (valor próprio de 4,117, variância de 16,4\%); presente-fatalista (valor próprio de 2,907, variância de 11,6\%); passado-positivo (valor próprio de 2,040, variância de 8,1\%); futuro (valor próprio de 1,735, variância de 6,9\%) e presente-hedonista (valor próprio de 1,460, variância de 5,8\%).

Após, foram verificadas as intercorrelações entre as subescalas do ZTPI-25 e analisadas as correlações das subescalas do ZTPI-25 com dados socioeconômicos e clínicos e com os domínios da qualidade de vida para a verificação da validade convergente (Tabela 3). 
Tabela 3 - Intercorrelações das subescalas do ZTPI-25 e correlações das subescalas do ZTPI-25 com dados socioeconômicos e clínicos e os domínios da qualidade de vida, Rio de Janeiro e Niterói, 2018.

\begin{tabular}{|c|c|c|c|c|c|}
\hline & $\mathbf{P N}$ & $\mathbf{P P}$ & PF & $\mathbf{P H}$ & $\mathbf{F}$ \\
\hline \multicolumn{6}{|l|}{ Intercorrelações } \\
\hline Passado-negativo & - & & & & \\
\hline Passado-positivo & 0,11 & - & & & \\
\hline Presente-fatalista & $0,27^{* *}$ & 0,10 & - & & \\
\hline Presente-hedonista & $0,28^{* *}$ & $0,20^{* *}$ & $0,34^{* *}$ & - & \\
\hline Futuro & $-0,09$ & $0,21^{* *}$ & $-0,02$ & 0,03 & - \\
\hline \multicolumn{6}{|l|}{ Correlações } \\
\hline Sexo (Masc/Fem) & 0,10 & $-0,04$ & $0,18^{* *}$ & $-0,07$ & $-0,04$ \\
\hline Idade & $-0,09$ & $-0,09$ & $0,12^{*}$ & $-0,18^{* *}$ & 0,01 \\
\hline Escolaridade & $-0,27^{* *}$ & 0,02 & $-0,24^{* *}$ & $-0,16^{* *}$ & 0,08 \\
\hline Usa TARV (Não/Sim) & 0,00 & $-0,12^{*}$ & $0,13^{*}$ & $-0,09$ & $-0,05$ \\
\hline Sintomas (Não/Sim) & $0,13^{*}$ & 0,02 & $0,13^{*}$ & 0,09 & $-0,10$ \\
\hline Físico & $-0,34^{* *}$ & 0,10 & $-0,09$ & $-0,09$ & $0,38^{* *}$ \\
\hline Psicológico & $-0,44^{* *}$ & $0,16^{* *}$ & $-0,13^{*}$ & $-0,08$ & $0,35^{* *}$ \\
\hline Nível de independência & $-0,28^{* *}$ & $0,13^{*}$ & $-0,13^{*}$ & $-0,06$ & $0,39^{* *}$ \\
\hline Relações sociais & $-0,22^{* *}$ & $0,18^{* *}$ & $-0,10$ & 0,00 & $0,28^{* *}$ \\
\hline Meio ambiente & $-0,27^{* *}$ & $0,16^{* *}$ & $-0,08$ & $-0,09$ & $0,31^{* *}$ \\
\hline Espiritualidade & $-0,34^{* *}$ & $-0,09$ & $-0,11^{*}$ & $-0,15^{* *}$ & $0,17^{* *}$ \\
\hline
\end{tabular}

${ }^{*} \mathrm{p} \leq 0,05 ;{ }^{* *} \mathrm{p} \leq 0,01$. Fonte: Autores (2018).

Os resultados da Tabela 3 apresentam padrão semelhante ao esperado, isto é, as intercorrelações entre passadonegativo, presente-fatalista e presente-hedonista, bem como passado-positivo e futuro se apresentam na direção esperada e significativas, indicando homogeneidade entre os fatores. As correlações também se comportam conforme o esperado, isto é, os menores escores de qualidade de vida têm correlação significativa com as subescalas negativas, passado-negativo e presente-fatalista, e os maiores escores de qualidade de vida apresentam correlação com as subescalas mais positivas, passadopositivo e futuro. De modo análogo, os dados socioeconômicos e clínicos, visto que a menor escolaridade se correlaciona com passado-negativo, presente-fatalista e hedonista e a presença de sintomas com passado-negativo e presente-fatalista.

A partir dos resultados encontrados, apresenta-se a versão curta do instrumento, ZTPI-25, proposta neste estudo na Figura 1, bem como as orientações de análise para utilização em outras pesquisas. 
Figura 1 - Versão curta do Inventário de Perspectiva Temporal de Zimbardo (ZTPI-25).

\section{Versão curta do Inventário de Perspectiva Temporal de Zimbardo (ZTPI-25)}

(Cecilio, Apostolidis, Lampropoulos, Oliveira, 2021)

Leia cada item e responda o mais sinceramente possível à pergunta: Em que medida esta característica é verdadeira para vocêe?

Para cada item assinale apenas uma alternativa. Por favor, responda todas as questões.

\begin{tabular}{|c|c|c|c|c|c|c|}
\hline & & $\begin{array}{l}\text { Nunca é } \\
\text { verdade }\end{array}$ & $\begin{array}{r}\text { Raramente } \\
\text { é verdade }\end{array}$ & Neutro & $\begin{array}{c}\text { Quase sempre } \\
\text { verdade }\end{array}$ & $\begin{array}{l}\text { Sempre } \\
\text { verdade }\end{array}$ \\
\hline 1(PP2) & $\begin{array}{l}\text { Imagens, sons e cheiros ligados à infância trazem muitas lembranças } \\
\text { maravilhosas. }\end{array}$ & 1 & 2 & 3 & 4 & 5 \\
\hline 2(PN4) & Penso frequentemente no que eu poderia ter feito diferente na minha vida. & 1 & 2 & 3 & 4 & 5 \\
\hline 3(PP7) & Sinto prazer ao pensar sobre meu passado. & 1 & 2 & 3 & 4 & 5 \\
\hline 4(PH8) & Faço as coisas impulsivamente. & 1 & 2 & 3 & 4 & 5 \\
\hline $5(\mathrm{~F} 10)$ & $\begin{array}{l}\text { Quando eu quero alcançar algo, determino metas e utilizo meios } \\
\text { específicos para alcançar esses objetivos. }\end{array}$ & 1 & 2 & 3 & 4 & 5 \\
\hline $6(\mathrm{PP} 11)$ & Em geral, tem muito mais coisas boas do que ruins no meu passado. & 1 & 2 & 3 & 4 & 5 \\
\hline 7(PN16) & Sempre me lembro de experiências passadas dolorosas. & 1 & 2 & 3 & 4 & 5 \\
\hline $8(\mathrm{PP} 20)$ & Boas memórias de tempos passados surgem facilmente na minha mente. & 1 & 2 & 3 & 4 & 5 \\
\hline $9(\mathrm{~F} 21)$ & Cumpro pontualmente com minhas obrigações com amigos e autoridades. & 1 & 2 & 3 & 4 & 5 \\
\hline 10(PH23) & Tomo minhas decisões no impulso do momento. & 1 & 2 & 3 & 4 & 5 \\
\hline 11(PF24) & Encaro cada dia como vier, ao invés de tentar planejá-lo. & 1 & 2 & 3 & 4 & 5 \\
\hline 12(PF28) & $\begin{array}{l}\text { Sinto que é mais importante aproveitar o que estou fazendo, do que fazer } \\
\text { o trabalho no tempo previsto. }\end{array}$ & 1 & 2 & 3 & 4 & 5 \\
\hline 13(PP29) & Sinto nostalgia (saudade) sobre a minha infância. & 1 & 2 & 3 & 4 & 5 \\
\hline 14(PH31) & Assumir riscos evita que minha vida seja entediante. & 1 & 2 & 3 & 4 & 5 \\
\hline 15(PF32) & $\begin{array}{l}\text { É mais importante para mim, curtir a vida do que alcançar objetivos } \\
\text { futuros. }\end{array}$ & 1 & 2 & 3 & 4 & 5 \\
\hline 16(PN34) & É difícil para eu esquecer lembranças desagradáveis da minha juventude. & 1 & 2 & 3 & 4 & 5 \\
\hline 17(PF37) & $\begin{array}{l}\text { Não podemos de fato planejar o futuro porque as coisas estão sempre } \\
\text { mudando. }\end{array}$ & 1 & 2 & 3 & 4 & 5 \\
\hline 18(PF39) & $\begin{array}{l}\text { Não faz sentido se preocupar com o futuro, já que não há nada mesmo que } \\
\text { se possa fazer. }\end{array}$ & 1 & 2 & 3 & 4 & 5 \\
\hline 19(F40) & $\begin{array}{l}\text { Termino meus projetos no tempo certo, avançando e progredindo em } \\
\text { ritmo constante. }\end{array}$ & 1 & 2 & 3 & 4 & 5 \\
\hline 20(PH42) & Eu me arrisco para ter excitação (emoção) na minha vida. & 1 & 2 & 3 & 4 & 5 \\
\hline $21(\mathrm{~F} 45)$ & Resisto a tentações quando sei que há trabalho a fazer. & 1 & 2 & 3 & 4 & 5 \\
\hline 22(PN50) & Penso nas coisas ruins que ocorreram comigo no passado. & 1 & 2 & 3 & 4 & 5 \\
\hline 23(PH53) & Muitas vezes a sorte funciona melhor do que o trabalho duro. & 1 & 2 & 3 & 4 & 5 \\
\hline 24(PN54) & Penso sobre as boas oportunidades que eu perdi na minha vida. & 1 & 2 & 3 & 4 & 5 \\
\hline $25(\mathrm{~F} 56)$ & Sempre terei tempo para colocar meu trabalho em dia. & 1 & 2 & 3 & 4 & 5 \\
\hline
\end{tabular}

*PN: passado-negativo; PP: passado-positivo; PF: presente-fatalista; PH: presente-hedonista; F: futuro.

Os números em parênteses correspondem ao item do ZTPI original.

Instruções de análise: inverter itens 11 e 25 , assim: "1" torna-se "5"; “2" torna-se "4"; "3" permanece "3"; "4" torna-se "2"; "5" torna-se "1". Após a codificação reversa dos itens, some as pontuações dos itens que compõem cada fator e divida pelo número de questões, isto é, por cinco. PN: somar itens 2, 7, 16, 22 e 24. Dividir por 5. PP: somar itens 1, 3, 6, 8 e 13. Dividir por 5. PF: somar itens 11, 12, 15, 17 e 18. Dividir por 5. PH: somar itens 4, 10, 14, 20 e 23. Dividir por 5. F: somar itens 5, 9, 19, 21 e 25. Dividir por 5.

Fonte: Autores (2018). 


\section{Discussão}

O teste KMO sugere bom ajuste, de acordo com os valores padrão, e o de Bartlett indica adequação da amostra e o uso da AFE como método pertinente (Figueiredo \& Silva, 2010). Quanto às propriedades psicométricas (valor próprio, variância e alfa de Cronbach), o Modelo 5 - ZTPI-25 - apresenta melhores índices que as demais versões brasileiras (Leite \& Pasquali, 2008; Milfont et al., 2008). O índice de variância para o Modelo 5 (49,0\%) foi superior ao índice da versão original americana (36\%) (Zimbardo \& Boyd, 1999) e superior a uma das versões brasileiras (33,3\%) (Leite \& Pasquali, 2008), o que indica a melhor explicação para o fenômeno da perspectiva temporal pelo ZTPI-25. O ZTPI-25 apresentou uma estrutura mais estável, visto que as questões foram reduzidas àquelas que melhor descrevem cada componente.

Um estudo apresentou a comparação entre validações do ZTPI em 24 países, apontando variâncias entre 29,9\% e 39,4\%, com média de 35,05\% (Sircova et al., 2014). Embora os valores encontrados tenham sido semelhantes, continua aberta a discussão sobre a explicação do fenômeno da perspectiva temporal, desde a versão original até as traduções em diferentes culturas (Apostolidis \& Fieulaine, 2004; Davis \& Ortiz, 2017; Sircova et al., 2014).

Portanto, evidencia-se que grande parte do fenômeno da perspectiva temporal permanece não explicada, reforçando a necessidade de desenvolvimento de novas pesquisas sobre a temática e a busca de melhores resultados para o ZTPI. Apesar da baixa variância, sabe-se da importância dessa construção psicológica para a compreensão dos comportamentos em saúde, desde a exposição às infecções sexualmente transmissíveis, como o HIV e a adesão a tratamentos, até a utilização de substâncias psicoativas, além da relação com ansiedade e depressão e, ainda, a associação com a qualidade de vida, já apresentados em estudos anteriores (Apostolidis \& Fieulaine, 2004; Fieulaine \& Martinez, 2010; Laguette et al., 2013; Préau et al., 2007; Zhang, Howell \& Bowerman, 2013; Zimbardo \& Boyd, 1999).

Embora alguns itens tenham apresentado cargas fatoriais satisfatórias em mais de um componente, reitera-se a importância de interpretar os fatores em apenas um, respaldado no pressuposto da AFE de revelar uma estrutura simples, garantindo que o mesmo item não seja interpretado em fatores distintos (Figueiredo \& Silva, 2010). Esse procedimento não foi observado no estudo de validação brasileira do Modelo 2 (Leite \& Pasquali, 2008), implicando na utilização de um mesmo item em mais de um componente, fragilizando o conjunto da escala. No presente estudo, mesmo com a redução, as médias das subescalas permaneceram semelhantes, possivelmente respondendo apenas a variação de itens. Os valores do alfa para o ZTPI25 foram próximos ao original e são satisfatórios (Brown, 2006; Zimbardo \& Boyd, 1999).

A AFC revelou resultados que permitem comparar os modelos de 1 a 5 . A análise dos índices definidos para identificação do melhor modelo mostra que o ZTPI-25 se apresenta ajustado aos dados. Considerando os quatro índices utilizados para identificar o melhor modelo, três (RMSEA, $\chi 2 / g 1$ e número de iterações) favorecem o Modelo 5 e um (CFI) o Modelo 4. Ressalta-se que a redução de fatores e itens não deve considerar apenas os ajustes estatísticos, mas também o refinamento da escala e a manutenção dos índices de confiabilidade e consistência interna, além das premissas teóricas do instrumento durante o processo de redução (McKay et al., 2015; Perry et al., 2015), que reforçam o ZTPI-25 como o melhor modelo para a população brasileira.

As intercorrelações entre as subescalas conferiram validade estrutural ao ZTPI-25 ao apresentar semelhanças com estudos anteriores, indicando a homogeneidade entre os fatores (Apostolidis \& Fieulaine, 2004; Milfont et al., 2008; Zimbardo \& Boyd, 1999). Ainda, a validade convergente foi admitida por meio das análises das correlações entre os dados socioeconômicos e clínicos das pessoas vivendo com HIV, os escores dos domínios da qualidade de vida e os escores das subescalas do ZTPI-25.

Quanto à correlação com os dados socioeconômicos e clínicos, os resultados sinalizam a validade do ZTPI-25, visto que a correlação entre sexo feminino e presente-fatalista foi anteriormente relatada, bem como as correlações das subescalas mais negativas (passado-negativo, presente-fatalista e hedonista) com a menor escolaridade e as subescalas do presente com 
idades mais jovens (Dany et al., 2015; Košt’ál et al., 2016; Milfont et al., 2008; Wakefild et al., 2010). As correlações entre o uso da terapia antirretroviral e a presença de sintomas sugerem pessimismo, negatividade e um possível descontrole frente às condições que fragilizam a saúde e corroboram com o conteúdo das subescalas do passado-negativo e presente-fatalista (Laguette et al., 2013).

Destarte, a subescala do passado-negativo deve se correlacionar com proposições que afirmam a presença de sentimentos negativos, baixa satisfação com a vida, entre outros. Tal relação foi confirmada pelas correlações negativas com todos os domínios da qualidade de vida, indicando que quanto maior o escore de passado-negativo, menor o escore de qualidade de vida (Boniwell et al., 2010; Laguette et al., 2013; Préau et al., 2007; Zhang, Howell \& Bowerman, 2013).

O passado-positivo corrobora achados relacionados ao seu efeito como protetor das práticas de saúde, identificado neste estudo pelas correlações positivas com alto escore de qualidade de vida nos domínios psicológico, nível de independência, relações sociais e meio ambiente (Laguette et al., 2013; Préau et al., 2007). O presente-fatalista, em estudos anteriores, foi associado com comportamento agressivo, ansiedade e depressão (Zimbardo \& Boyd, 1999). Esta relação foi verificada neste estudo na correlação negativa para os domínios psicológico, nível de independência e espiritualidade.

A subescala presente-hedonista reflete o viver com prazer, sendo comumente correlacionada com o uso de substâncias psicoativas (Fieulaine \& Martinez, 2010). Neste sentido, a correlação negativa com o domínio espiritualidade, religião e crenças pessoais, confirma a tendência de assumir riscos e não se preocupar com as consequências futuras. $\mathrm{O}$ futuro confirma sua validade ao correlacionar-se positivamente com todos os domínios da qualidade de vida, visto que geralmente se associa a maior preocupação com a saúde, estilo de vida saudável e atitudes conscientes (Milfont et al., 2008).

Considerando a aplicação do construto no campo da saúde e da enfermagem, a incorporação da avaliação da perspectiva temporal nas ações de cuidado à saúde também pode contribuir na prática da assistência, tanto para prevenção de comportamentos considerados danosos diante de uma condição patológica, quanto para o desenvolvimento de perspectivas reforçadoras de comportamentos protetores (Oliveira, Cecilio \& Oliveira, 2020).

\section{Conclusão}

Desde o desenvolvimento do ZTPI seu uso tem sido efetivo, sendo considerado como a mais completa e principal medida de tempo disponível na literatura. O ZTPI-25, proposto neste estudo, conserva solidamente as propriedades psicométricas da versão original e apresenta vantagens em relação a ela. Essas vantagens são: proposta simplificada, menor tempo de aplicação, menores índices de rejeição ou interrupção de respostas antes da finalização e redução de questões sem respostas ou com respostas não consistentes. Assim, recomenda-se a utilização do ZTPI-25 para a identificação dos construtos da perspectiva temporal, visto que os resultados apontam boas propriedades psicométricas e atestam a sua validade.

As limitações estão relacionadas à amostra de conveniência, o que restringe a possibilidade de generalização dos achados, além da necessidade de verificação da validade externa. Entretanto, ressalta-se que a amostra foi composta por pessoas atendidas em serviços públicos de saúde, em cidades com características sociodemográficas diferentes, o que conferiu variabilidade social, econômica e de escolaridade, o que não foi observado em outros estudos brasileiros de validação realizados com grupos homogêneos.

Quanto às potencialidades dos resultados, reforça-se a ideia de um instrumento único, simples, de fácil aplicação que pode ser amplamente utilizado em pesquisas nas diversas áreas do conhecimento. Sendo assim, sugere-se a aplicação do ZTPI25 em outras populações e contextos, a fim de aprimorar sua validade. Isto poderá permitir o desenvolvimento da teoria da perspectiva temporal, com ampla aplicação para a compreensão dos fenômenos psicológicos e de saúde. 


\section{Referências}

Apostolidis, T., \& Fieulaine, N. (2004). Validation française de l'échelle de temporalité The Zimbardo Time Perspective Inventory (ZTPI). Revue Europeenne de Psychologie Appliquee, 54, 207-217.

Boniwell, I., Osin, E., Alex Linley, P., \& Ivanchenko, G. V. (2010). A question of balance: Time perspective and well-being in British and Russian samples. The Journal of Positive Psychology, 5(1), 24-40.

Brown, T. A. (2006). Confirmatory Factor Analysis for Applied Research. The Guilford Press.

Cecilio, H. P. M., Oliveira, D. S., Apostolidis, T., Dany, L., \& Oliveira, D. C. (2020). Perspectiva temporal, status socioeconômico e qualidade de vida de pessoas vivendo com HIV. Research, Society and Development, 9(8), e148985451.

Dany, L., Roussel, P., Laguette, V., Lagouanelle-Simeoni, M. C., \& Apostolidis, T. (2015). Time perspective, socioeconomic status, and psychological distress in chronic pain patients. Psychol Health Med, 21(3), 295-308.

Davis, M. A., \& Ortiz, D. A. (2017). Revisiting the structural and nomological validity of the Zimbardo time perspective inventory. Personality and Individual Differences, 104, 98-103.

Fieulaine, N., \& Martinez, F. (2010). Time under control: Time perspective and desire for control in substance use. Addictive Behaviors, 35(8), 799-802.

Figueiredo, D. B., \& Silva, J. A. (2010). Visão além do alcance: uma introdução à análise fatorial. Opinião Pública, 16(1), 160-185.

Košt’ál, J., Klicperová-Baker, M., Lukavská, K., \& Lukavský, J. (2016). Short version of the Zimbardo Time Perspective Inventory (ZTPI-short) with and without the Future-Negative scale, verified on nationally representative samples. Time \& Society, 25(2), 169-192.

Laguette, V., Apostolidis, T., Dany, L., Bellon, N., Grimaud, J. C., \& Lagouanelle-Simeoni, M. C. (2013). Quality of life and time perspective in inflammatory bowel disease patients. Quality of Life Research, 22(10), 2721-2736.

Laros, J. A. (2012). O uso da análise fatorial: algumas diretrizes para pesquisadores. In: Pasquali L. (Eds.), Análise fatorial para pesquisadores. Brasília (DF): LabPAM Saber e Tecnologia.

Leite, U. R., \& Pasquali, L. (2008). Estudo de validação do Inventário de Perspectiva de Tempo do Zimbardo. Avaliação Psicológica, 7(3), 301-320.

Lewin K. Field Theory of Social Science: Selected Theoretical Papers. New York (NY): Harper \& Brothers, 1951.

McKay, M. T., Worrell, F. C., Temple, E. C., Perry, J. L., Cole, J. C., \& Mello, Z. R. (2015). Less is not always more: The case of the 36-item short form of the Zimbardo Time Perspective Inventory. Personality and Individual Differences, 72, 68-71.

Milfont, T. L., Andrade, P. R., Belo, R. P., \& Pessoa, V. S. (2008). Testing Zimbardo Time Perspective Inventory in a Brazilian sample. Interamerican Journal of Psychology, 42(1), 49-58.

Nuttin J, Lens W. Future time perspective and motivation. Theory and research method. Bélgica: Leuven University Press/Hillsdale, 1985.

Oliveira, D. S., Cecilio, H. P. M., \& Oliveira, D. C. (2020). Perspectiva temporal: discussões sobre a aplicação do conceito na área da saúde e na enfermagem. Rev Enferm UERJ. 28, e40392.

Organização Mundial da Saúde - OMS. (2002). Instrumento WHOQOL-HIV: sintaxe. Departamento de Saúde Mental e Dependência Química. Genebra: OMS.

Orkibi, H. (2015). Psychometric Properties of the Hebrew Short Version of the Zimbardo Time Perspective Inventory. Evaluation \& the Health Professions, $38(2), 219-245$.

Ortuño, V. E. C., Janeiro, I. N., Cordeiro, P., Paixão, M. P., \& Gamboa, V. (2017). Inventário de Perspectiva Temporal de Zimbardo (IPTZ). In L. S. Almeida, M. R. Simões, \& M. M. Gonçalves (Orgs.), Adaptação, desenvolvimento e sucesso acadêmico dos estudantes do ensino superior: instrumentos de avaliação (pp. 80-94) ADIPSIEDUC: Braga.

Perry, J. L., McKay, M. T., Worrell, F. C., Živkovič, U., Mello, Z. R., \& Musil, B. (2015). Measuring time perspective in adolescents: Can you get the right answer by asking the wrong questions? Personality and Individual Differences, 78, 53-57.

Préau, M., Apostolidis, T., Francois, C., Raffi, F., \& Spire, B. (2007). Time perspective and quality of life among HIV-infected patients in the context of HAART. AIDS Care, 19(4), 449-458.

Roncancio, A. M., Ward, K. K., \& Fernandez, M. E. (2014). The influence of time perspective on cervical cancer screening among Latinas in the United States. J Health Psychol, 19(12), 1547-1553.

Sircova, A., van de Vijver, F. J. R., Osin, E., Milfont, T. L., Fieulaine, N., \& Kislali-Erginbilgic, A., et al. (2014). A Global Look at Time: A 24-Country Study of the Equivalence of the Zimbardo Time Perspective Inventory. SAGE Open, 4(1),1-12.

Wakefield, C. E., Homewood , J., Taylor, A., Mahmut, M., \& Meiser, B. (2010). Time Perspective in Hereditary Cancer: Psychometric Properties of a Short Form of the Zimbardo Time Perspective Inventory in a Community and Clinical Sample. Genet Test Mol Biomarkers, 14(5), 617-627.

Zhang, J. W., Howell, R. T., \& Bowerman, T. (2013). Validating a brief measure of Zimbardo Time Perspective Inventory. Time \& Society, 22(3), 391-409.

Zimbardo, P. G., \& Boyd, J. N. (1999). Putting Time in Perspective: A Valid, Reliable Individual-Differences Metric. Journal of Personality and Social Psychology, 77(6), 1271-1288. 
Research, Society and Development, v. 10, n. 2, e43410212776, 2021

(CC BY 4.0) | ISSN 2525-3409 | DOI: http://dx.doi.org/10.33448/rsd-v10i2.12776

Zimpel, R. R., \& Fleck, M. P. (2007). Quality of life in HIV-positive Brazilians: application and validation of the WHOQOL-HIV, Brazilian version. AIDS Care, 19(7), 923-930. 